\title{
Change in motor cortex activation for muscle release by motor learning
}

\author{
Kenichi SugAwARA, RPT, Ph.D. \\ School of Rehabilitation, Faculty of Health and Social Services, Kanagawa University of Human Services
}

\begin{abstract}
For central nervous system disorders' rehabilitation, it is important to accurately understand motor control and implement an appropriate motor learning process to induce neuroplastic changes. The neurophysiological studies have revealed that neural control mechanisms are crucial during both the onset of muscular activities and muscle release after contraction. When performing various movements during daily activities, muscle relaxation control enables precise force output and timing control. Moreover, surround inhibition is a functional mechanism in the motor system. Surround inhibition of the motor system may be involved in the selective execution of desired movements. This review demonstrates cortical excitability resulting from motor learning, movement control mechanisms including muscle relaxation and the suppression of nontarget muscle groups, and the voluntary drive's importance that is required for movement. Key words: motor learning, muscle relaxation, surround inhibition, transcranial magnetic stimulation
\end{abstract}

(Phys Ther Res 23: 106-112, 2020)

F or physical therapists, it is crucial to understand the neural mechanisms of motor learning and motor control during daily activities. There are multiple factors that are involved in the selection of appropriate motor tasks to perform specific movements. Motor learning is responsible for improving various movement factors (e.g., speed and switching between skills) by adopting specific training methods based on a cognitive approach. This involves the learning of sensorimotor associations of various circuits used to perform specific tasks ${ }^{1)}$. The sensorimotor integration involves the input of sensory information and its conversion into movement to adapt to alterations in the environment. Moreover, forward models in motor learning help predict changes in the body's state during movement, which happen at a faster rate than sensorimotor integration ${ }^{2}$.

There is a significant connection between motor learning and the changes in central nervous system processing, and skillful motor learning depends on the plasticity of the primary motor cortex (M1). The plasticity of the central

Received: August 6, 2020

Accepted: September 17, 2020

Advance Publication by J-STAGE: December 4, 2020

Correspondence to: Kenichi Sugawara, School of Rehabilitation, Faculty of Health and Social Services, Kanagawa University of Human Services, 1-10-1 Heisei-cho, Yokosuka, Kanagawa 238-8522, Japan \# e-mail: sugawara-k@kuhs.ac.jp

doi: 10.1298/ptr.R0010 nervous system includes changes in the excitability of the M1 representation area for the specific muscle groups associated with a movement. As a result, the representation of movements in corticospinal tract cells is enhanced. Furthermore, other neural connections and changes in the plasticity of various interneurons occur.

It has been well acknowledged that motor skill learning modifies the central nervous system, especially the corticomotoneuronal system ${ }^{3)}$. These processes have been studied with the use of various cortical imaging techniques, including transcranial magnetic stimulation (TMS $)^{4)}$, functional magnetic resonance imaging ${ }^{5)}$, and electroencephalography $^{6}$, among others ${ }^{7,8}$. The motor control mechanism in the expression of voluntary movements is also considered an important research subject. Several studies have assessed central nervous system excitability following motor learning using motor-evoked potential (MEP) and H-reflex for the upper or lower central nervous system, respectively.

In the M1 of patients with multiple disorder types, the motor learning process leads to improved performance and characteristic excitability changes ${ }^{9,10}$. Previous TMS studies revealed that there is an association between improvements in motor performance and the central reorganization of specific muscles involved in skilled movement tasks ${ }^{11-15}$.

In physical therapy, the main aim for patients is to achieve a correct movement. As such, it is important to accumulate verified knowledge concerning control dynamics 
of all movement types. Moreover, acquiring an applied perspective on the motor-neurophysiological processes depends on creating an unwavering foundation for its development in physical therapy.

To date, there have been several studies on cortical excitability following various voluntary movements. We focus in this review on cortical excitability arising from motor learning, the control of muscle relaxation or suppression of nontarget muscle groups, and the importance of voluntary muscle release that is required for movement.

\section{Muscle Relaxation in Motor Learning}

The motor control of muscle relaxation aids movement and the achievement of precise force output and timing control $^{16-19)}$. There are multiple central nervous system disorders, including stroke, Parkinson's disease, and dystonia, which are related to motor control ${ }^{20-24)}$.

The mechanisms of the motor cortex in voluntary muscle relaxation have been investigated using TMS and other techniques in clinical neurophysiological studies ${ }^{25,26}$. However, these muscle relaxation studies showed confusing results. For example, Begum et al. ${ }^{25)}$ reported that shortinterval intracortical inhibition ( SICI) decreased before muscle relaxation. Conversely, M1 excitability decreased and SICI increased before and at the onset of relaxation ${ }^{27}$. The conflicting observations may be due to the assessment of varying relaxation strategies. Moreover, Motawar et al. ${ }^{26)}$ revealed that different TMS strategies were used in the two studies and suggested that SICI increased before muscle relaxation and gradually increased as muscle relaxation progressed. Furthermore, at the start of muscle relaxation, the underlying central nervous system mechanisms may be different from those at the onset of voluntary muscle contraction.

The development of clinical rehabilitation strategies for muscle relaxation in affected patients is crucial to enable them to control muscle contraction or relaxation through appropriate motor training. Importantly, many patients with central nervous system disorder experience symptoms such as paretic limb muscles, including spasticity and loss of sensation, among others. Therefore, appropriate motor learning methods for improving the control of voluntary muscle release may be useful for disabled patients attempting to perform accurate movements. Efforts have been made to improve muscle relaxation with motor learning techniques, resulting in increased motor cortex excitability in the antagonist muscle ${ }^{28}$. Furthermore, there is a need to investigate the motor cortex excitability with motor learning during muscle release further.

\section{The Functional Meaning of Surround Inhibition by Motor Training}

Cortical surround inhibition (SI) suppresses neural circuit excitability of the motor and sensory cortex, thus enabling neural activity to focus on the appropriate motor response. The SI functional meaning is to provide spatiotemporal discrimination of various sensory inputs in the sensory cortex ${ }^{29}$. Furthermore, SI is identified as an essential mechanism of the motor output system. Cortical SI of the motor system is potentially involved in carrying out required movements and inhibition of the unwanted movements of muscle groups ${ }^{30)}$.

Additionally, SI in the motor system has been defined as an adjustment in the $\mathrm{M}^{31,32)}$, and damage to SI in the motor system may cause focal hand dystonia ${ }^{33-36)}$ and parkinsonism $^{37)}$. In these reports, to prevent the activation of unwanted muscle activities, TMS was used during volatile movements and the authors observed that SI resulted in increased activity of the SICI circuit ${ }^{38)}$. In another study, it has also been indicated that SI is suppressed at the supraspinal level and constitutes a significant principle of the motor output system $^{32)}$. Furthermore, the modulation of SICI circuits in the motor cortex potentially enhances the required movements by decreasing the selected motor output pathway inhibition $^{39,40)}$. Moreover, deficient SI has been observed in various neural disorders and was shown to be modulated by the task difficulty ${ }^{41)}$ and hemispheric asymmetry ${ }^{42)}$. As mentioned previously, we will explain in this review the mechanisms behind motor control during the suppression of nontarget muscle groups by using an example of a neurophysiological experimental method as revealed by neurophysiological experiments using TMS and H-reflex.

\section{The Demonstration of Cortical Excitability in Relaxation Mechanisms after Motor Learning}

In this review, we used the tracking method to examine modifications in motor cortex excitability before and after motor learning at high and low motor output levels during ramp muscle relaxation ${ }^{43)}$. As shown in Figure 1, this method intends to record muscle release reflected on the computer screen. The subjects were asked to perform controlled wrist extension at $30 \%$ maximum voluntary contraction (MVC) for $2 \mathrm{~s}$, from the $30 \%$ MVC to ramp muscle release (Fig. 1). Referring to the computer screen, the dots were measured by a force transducer as it moved down along the ramp waveform (Fig. 1). Using this method, whether M1 excitability depended on muscle relaxation after motor learning was investigated. The motor training consisted of 10 sets, and each set was composed of 10 trials. MEPs by TMS from the extensor and flexor carpi radialis (ECR), agonist muscle, and flexor carpi radialis 


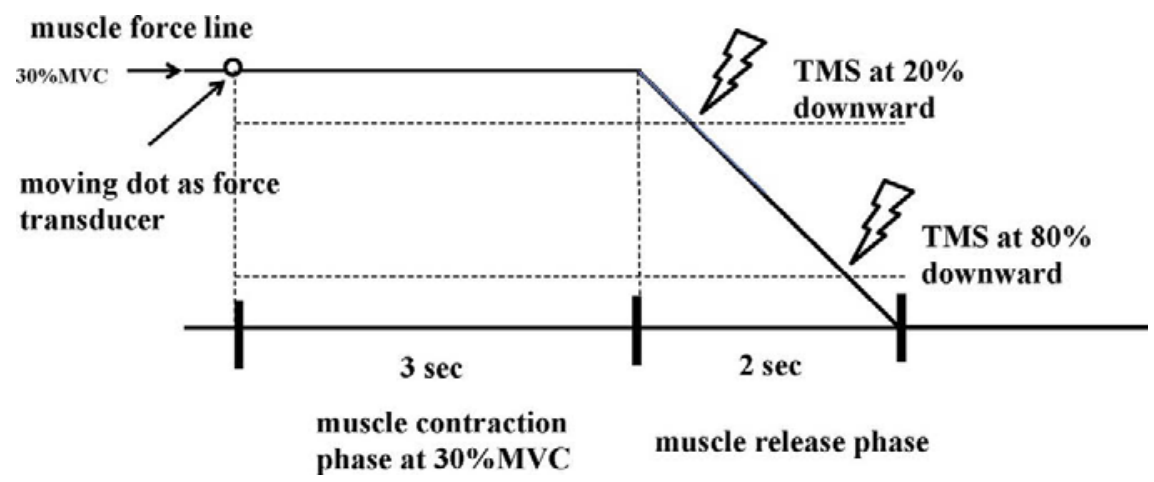

Figure 1. Experimental setup for measuring the extensor carpi radialis (ECR) and flexor carpi radialis (FCR) at $20 \%$ or $80 \%$ of the downward force output from $30 \%$ of maximum voluntary contraction (MVC) and the timing of the transcranial magnetic stimulation (TMS) pulses (Sugawara et al., 2016 38) ).

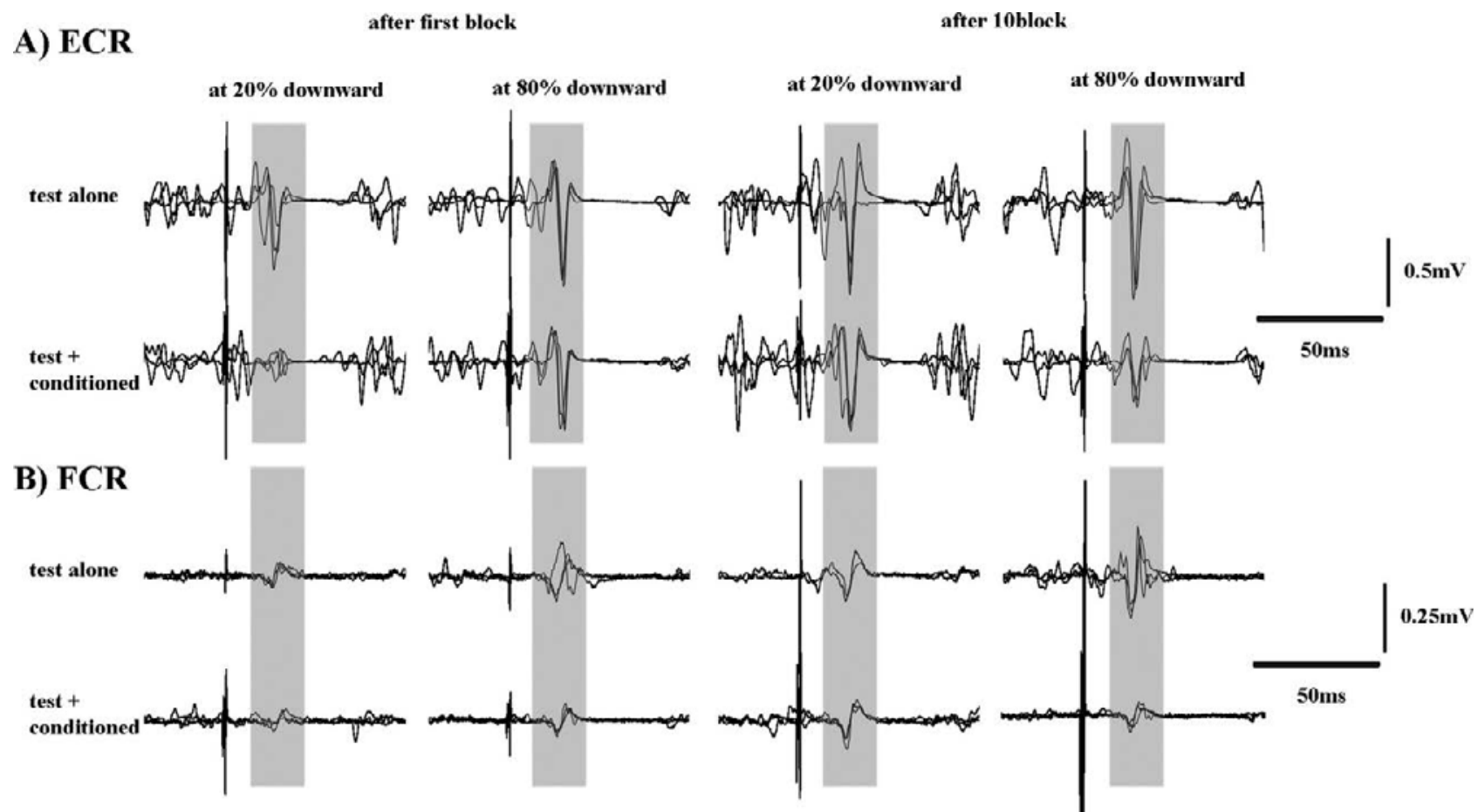

Figure 2. Sample motor-evoked potential (MEP) recordings from (A) the extensor carpi radialis (ECR) and (B) flexor carpi radialis (FCR) muscles obtained from a single subject in response to single- and paired-pulse transcranial magnetic stimulation (TMS) at $20 \%$ or $80 \%$ of the downward force output during muscle relaxation (Sugawara et al., 2016 38) ).

(FCR), antagonist muscle were subsequently measured and compared to evaluate the states "before" and "after" motor learning. TMS was delivered during muscle relaxation at $20 \%$ or $80 \%$ of the muscle force output from $30 \%$ MVC as shown in Figure 1. TMS was delivered via single- or paired-pulse TMS trials, which were randomly delivered 20 times at each of the two conditions (at $20 \%$ and $80 \%$ ). The MEP and SICI values in these two phases - at $20 \%$ and $80 \%$ - were compared in each agonist (ECR) and antagonist (FCR) immediately after the 1 st and 10th blocks. During muscle relaxation in both muscles and downward force output, motor cortex excitability increased significantly after motor learning (Fig. 2). Additionally, in the agonist muscle (ECR), the SICI after motor learning increased significantly during the $80 \%$ waveform decline when compared to before motor learning (Fig. 2). In the antagonist muscle (FCR), the SICI also exhibited a greater inhibitory effect when muscle relaxation was terminated (80\% downward) after motor learning (Fig. 2). During motor training, acquisition of the ability to control muscle relaxation increased the SICI in both the ECR and FCR during motor termination.

As indicated by the imaging studies, neural activation of voluntary muscle relaxation has been observed in the motor cortex and supplementary motor areas before release and during release from the muscle contraction phase ${ }^{44-46)}$. 


\section{A. Background EMG}

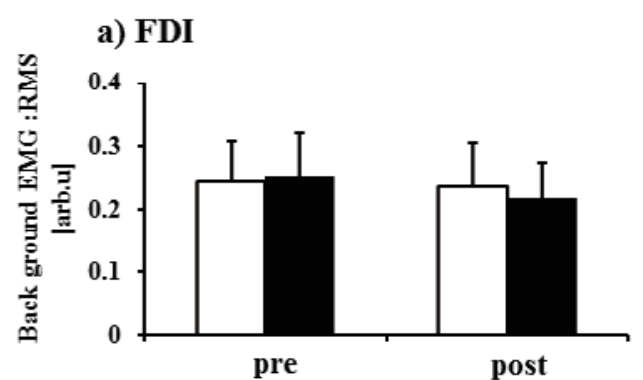

B. MEP

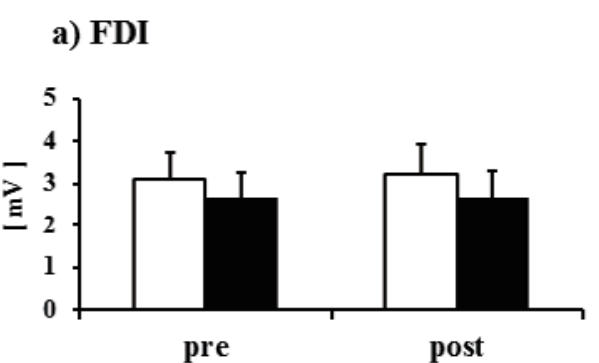

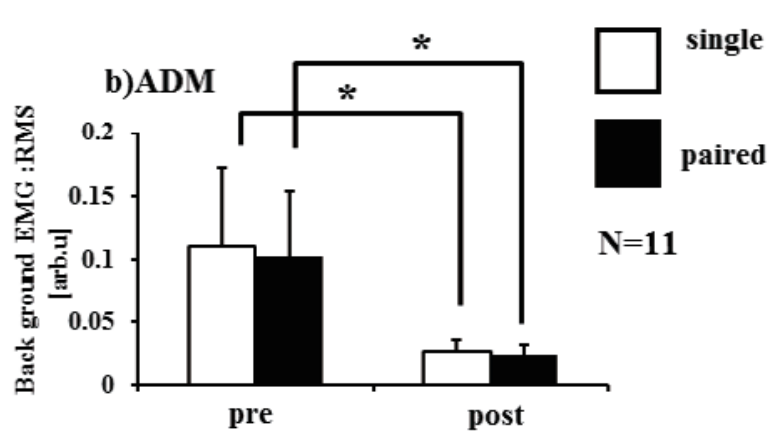

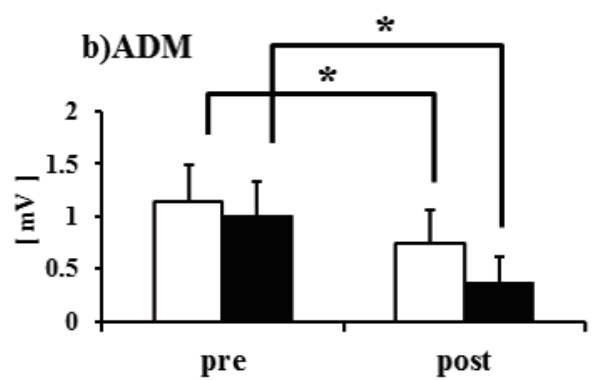

Figure 3. (A) Mean $( \pm$ SE) background electromyographic (EMG) activity of (a) first dorsal interosseous (FDI) and (b) abductor digiti minimi (ADM) muscles before (pre) and after (post) the training $(N=$ 11). Background EMG activity of the FDI muscle was unchanged by the training, whereas background EMG activity from ADM was reduced after the training $(* P<0.05)$. (B) Mean motorevoked potentials (MEPs) from (a) FDI and (b) ADM before and after the training. Similar to the results observed for the background EMG activities, MEPs from the FDI muscle were unchanged by the training, whereas MEPs from the ADM muscle reduced significantly after the training. RMS: root mean square (Sugawara et al., 2012 48) ).

The motor cortex is activated by the onset of muscle relaxation and contraction, but results show the output of different movements ${ }^{45-47)}$. On the other hand, the H-reflex does not show active changes during muscle relaxation at the spinal level ${ }^{48-51}$. Moreover, we have confirmed that the M1 excitability increase is observed just before muscle relaxation, which indicates that the increased excitability is significant in relaxation after muscle contraction ${ }^{52)}$. The ongoing excitatory input to M1 is inhibited by the offset of isometric contraction ${ }^{47)}$. Furthermore, it has been suggested that cortical inhibitory activation plays an important role in muscle relaxation ${ }^{26)}$. Moreover, neural modifications in the M1 during muscle release reveal that the central mechanisms underlying muscle relaxation are induced and achieved by active control and do not occur automatically $^{44)}$. These findings revealed that motor control of muscle release possibly uses specific neural excitability of the motor cortex and inhibitory neural function. Finally, understanding the mechanisms underlying muscle relaxation can result in achieving the desired motor output after motor learning.

\section{The SI Mechanisms after Motor Learning}

The suppression of nontarget muscle groups after mo- tor learning is required to study the mechanisms of $\mathrm{SI}^{53)}$. Hence, subjects were asked to perform sustained $40 \%$ MVC of the index finger abduction by first dorsal interosseous (FDI) with the abductor digiti minimi (ADM) relaxed. Particularly, we focused on the decrease in overflow electromyographic activity in ADM by motor training. When the participants fulfilled the criteria in ADM activities (less than 5\% MVC), the training was terminated. MEPs of single TMS and paired-pulse TMS for SICI were recorded simultaneously for both the ADM and FDI while maintaining index finger abduction at $40 \%$ MVC or during only motor imagery at $40 \%$ MVC before and after training, respectively.

The MEP and SICI of FDI remained unchanged after the training. In contrast, the MEP of the ADM reduced and the SICI circuits in the ADM were significantly strengthened after training. These findings were observed during both actual contraction and motor imagery after the FDI training (Fig. 3 and 4).

To control the suppressed ADM muscle, isolated activation of the FDI muscle may require enhanced SICI. It has been reported that unwanted ADM muscle activation during muscle contraction modulated the SICI, which indicates a preventive mechanism ${ }^{38,54)}$.

Moreover, results from a previous report did not sup- 
A. Specimen record

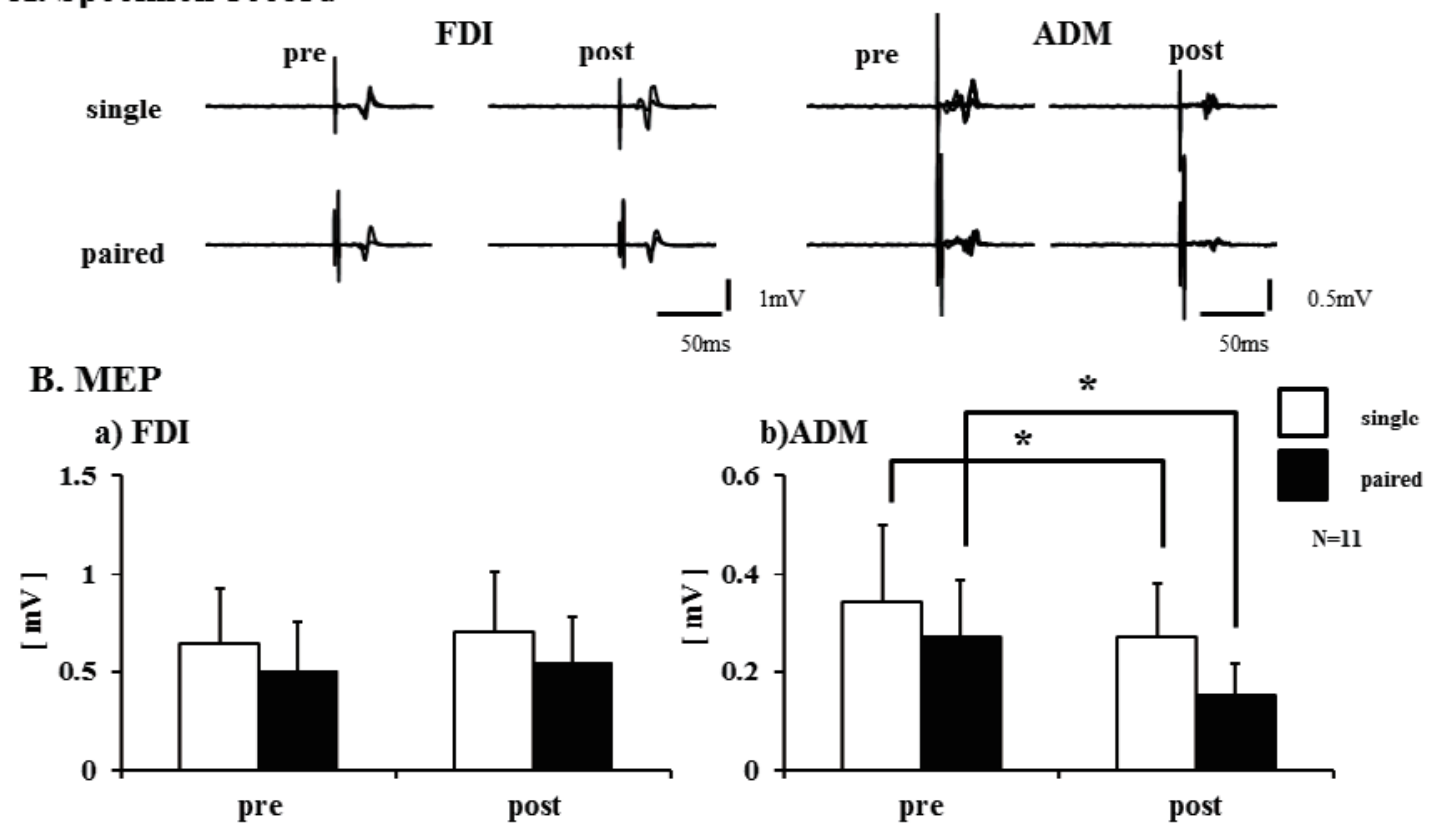

Figure 4. (A) Sample motor-evoked potential (MEP) recordings from the first dorsal interosseous (FDI) and abductor digiti minimi (ADM) muscles in response to single- and paired-pulse transcranial magnetic stimulation (TMS) during motor imagery of the index finger abduction. (B) Mean $( \pm \mathrm{SE})$ MEPs in response to single- and paired-pulse TMS before and after the training $(N=11)$. MEPs from the (a) FDI muscle were unchanged after the training, whereas MEPs from the (b) ADM muscle were decreased after the training, similar to the results obtained during the actual movement $(* P<0.05)\left(\right.$ Sugawara et al., $\left.2012{ }^{48)}\right)$.

port the involvement of SICI to $\mathrm{SI}^{33,35)}$. According to that study, the differences in motor tasks would be a cause of contradiction in the effect of SICI. Specifically, suppression of the unwanted muscle contraction was observed during the motor task. However, the present review aimed to determine whether the unwanted ADM muscles are regulated by SI. This functional regulation was required for the skillful motor control execution.

Generally, the complex motor output of the hand often needed coordinated stabilizing and moving action, temporally and spatially, of many intrinsic and extrinsic hand muscles ${ }^{55-57)}$. The functional participation of the necessary stabilizing effect of the other hand muscles and the involuntary activation of other digits increase simultaneously when the level of force output by a single digit is increased ${ }^{57)}$. In our study, motor training was used to suppress the participation of the accompanying ADM muscle activity.

It is crucial to appropriately select the target muscle group in motor learning in reduced local inhibitory systems. Moreover, motor learning requires the suppression of unwanted muscle groups through enhanced $\mathrm{SICI}^{40)}$. The SICI circuits mediating SI were altered following motor learning. Therefore, it is suggested that the functional enhancement of SI controls unwanted movements.

\section{Conclusion}

In this review, we summarized the available information on motor cortex excitability with muscle release and focused, in particular, on motor learning. Motor cortex excitability is thought to be affected by the variation of motor characteristics such as relaxation from muscle contraction and SI by motor learning. Therefore, we performed several experiments involving single- or paired-pulse TMS studies.

Brain plasticity is considered a fundamental mechanism of learning and memory. Central nervous system plasticity, deeply involved in motor learning, ensures a flexible connection between neural networks and is a phenomenon reflecting its characteristics. In humans, MEP using this TMS is widely used as an index for grasping plastic changes in the motor cortex. In any case, TMS requires a very delicate technique, so it is necessary to gain sufficient training before working with the equipment and indicators.

Acknowledgments: This work was supported by a Grant-in-Aid for Scientific Research (C) 17 K01520 from the Japan Society for the Promotion of Science. In addition, the author would like to thank Editage (https://www.editag e.jp) for editorial assistance with the manuscript.

Conflict of Interest: The author declares no conflicts of 
interest.

\section{References}

1) Makino H, Hwang EJ, et al.: Circuit Mechanisms of Sensorimotor Learning. Neuron. 2016; 92: 705-721.

2) Scott SH: A Functional Taxonomy of Bottom-Up Sensory Feedback Processing for Motor Actions. Trends Neurosci. 2016; 39: 512-526.

3) Sugawara K, Tanabe S, et al.: Different motor learning effects on excitability changes of motor cortex in muscle contraction state. Somatosens Mot Res. 2013; 30: 133-139.

4) Ljubisavljevic $M$ : Transcranial magnetic stimulation and the motor learning-associated cortical plasticity. Exp Brain Res. 2006; 173: 215-222.

5) Gonzalez CC and Burke MR: Motor Sequence Learning in the Brain: The Long and Short of It. Neuroscience. 2018; 389: 8598.

6) Wu J, Knapp F, et al.: Electroencephalographic connectivity measures predict learning of a motor sequencing task. J Neurophysiol. 2018; 119: 490-498.

7) Boonstra TW, Daffertshofer A, et al.: Multivariate timefrequency analysis of electromagnetic brain activity during bimanual motor learning. Neuroimage. 2007; 36: 370-377.

8) Sugawara K, Onishi H, et al:: Repeated practice of a Go/NoGo visuomotor task induces neuroplastic change in the human posterior parietal cortex: an MEG study. Exp Brain Res. 2013; 226: 495-502.

9) Karni A, Meyer G, et al.: Functional MRI evidence for adult motor cortex plasticity during motor skill learning. Nature. 1995; 377: 155-158.

10) Nudo RJ, Milliken GW, et al.: Use-dependent alterations of movement representations in primary motor cortex of adult squirrel monkeys. J Neurosci. 1996; 16: 785-807.

11) Classen J, Liepert J, et al.: Rapid plasticity of human cortical movement representation induced by practice. J Neurophysiol. 1998; 79: 1117-1123.

12) Jensen JL, Marstrand PC, et al.: Motor skill training and strength training are associated with different plastic changes in the central nervous system. J Appl Physiol (1985). 2005; 99: 15581568.

13) Lotze M, Braun C, et al.: Motor learning elicited by voluntary drive. Brain. 2003; 126: 866-872.

14) Muellbacher W, Ziemann U, et al.: Role of the human motor cortex in rapid motor learning. Exp Brain Res. 2001; 136: 431438.

15) Perez MA, Lungholt BK, et al.: Motor skill training induces changes in the excitability of the leg cortical area in healthy humans. Exp Brain Res. 2004; 159: 197-205.

16) Kimura $\mathrm{T}$ and Gomi $\mathrm{H}$ : Temporal development of anticipatory reflex modulation to dynamical interactions during arm movement. J Neurophysiol. 2009; 102: 2220-2231.

17) Kutz DF, Wolfel A, et al.: Detection of changes in grip forces on a sliding object. J Neurosci Methods. 2007; 166: 250-258.

18) Nowak DA, Grefkes C, et al.: Dexterity is impaired at both hands following unilateral subcortical middle cerebral artery stroke. Eur J Neurosci. 2007; 25: 3173-3184.

19) Nowak DA, Hermsdorfer J, et al.: Deficits of predictive grip force control during object manipulation in acute stroke. J Neurol. 2003; 250: 850-860.

20) Kamper DG, Harvey RL, et al.: Relative contributions of neural mechanisms versus muscle mechanics in promoting finger extension deficits following stroke. Muscle Nerve. 2003; 28: 309318.

21) Seo NJ, Rymer WZ, et al.: Delays in grip initiation and termination in persons with stroke: effects of arm support and active muscle stretch exercise. J Neurophysiol. 2009; 101: 3108-3115.

22) Grasso M, Mazzini L, et al.: Muscle relaxation in Parkinson's disease: a reaction time study. Mov Disord. 1996; 11: 411-420.

23) Buccolieri A, Avanzino L, et al.: Muscle relaxation is impaired in dystonia: a reaction time study. Mov Disord. 2004; 19: 681687.

24) Yazawa S, Ikeda A, et al:: Abnormal cortical processing of voluntary muscle relaxation in patients with focal hand dystonia studied by movement-related potentials. Brain. 1999; 122( Pt 7): 1357-1366.

25) Begum T, Mima T, et al.: Cortical mechanisms of unilateral voluntary motor inhibition in humans. Neurosci Res. 2005; 53: 428435.

26) Motawar B, Hur P, et al.: Contribution of intracortical inhibition in voluntary muscle relaxation. Exp Brain Res. 2012; 221: 299308.

27) Buccolieri A, Abbruzzese G, et al.: Relaxation from a voluntary contraction is preceded by increased excitability of motor cortical inhibitory circuits. J Physiol. 2004; 558: 685-695.

28) Yoshida N, Yamaguchi T, et al.: Increasing corticospinal excitability in the antagonist muscle during muscle relaxation with a tracking task. Somatosens Mot Res. 2015; 32: 39-43.

29) Blakemore C, Carpenter RH, et al.: Lateral inhibition between orientation detectors in the human visual system. Nature. 1970; 228: 37-39.

30) Mink JW: The basal ganglia: focused selection and inhibition of competing motor programs. Prog Neurobiol. 1996; 50: 381-425.

31) Hallett M: Dystonia: abnormal movements result from loss of inhibition. Adv Neurol. 2004; 94: 1-9.

32) Sohn YH and Hallett M: Surround inhibition in human motor system. Exp Brain Res. 2004; 158: 397-404.

33) Sohn YH and Hallett M: Disturbed surround inhibition in focal hand dystonia. Ann Neurol. 2004; 56: 595-599.

34) Stinear CM and Byblow WD: Impaired modulation of intracortical inhibition in focal hand dystonia. Cereb Cortex. 2004; 14 : 555-561.

35) Beck S, Richardson SP, et al.: Short intracortical and surround inhibition are selectively reduced during movement initiation in focal hand dystonia. J Neurosci. 2008; 28: 10363-10369.

36) Richardson SP, Bliem B, et al.: Changes in short afferent inhibition during phasic movement in focal dystonia. Muscle Nerve. 2008; 37: 358-363.

37) Shin HW, Kang SY, et al.: Disturbed surround inhibition in pre- 
clinical parkinsonism. Clin Neurophysiol. 2007; 118: 21762179.

38) Sohn YH, Wiltz K, et al.: Effect of volitional inhibition on cortical inhibitory mechanisms. J Neurophysiol. 2002; 88: 333-338.

39) Stinear CM and Byblow WD: Role of intracortical inhibition in selective hand muscle activation. J Neurophysiol. 2003; 89: 2014-2020.

40) Zoghi M, Pearce SL, et al.: Differential modulation of intracortical inhibition in human motor cortex during selective activation of an intrinsic hand muscle. J Physiol. 2003; 550: 933-946.

41) Beck S and Hallett M: Surround inhibition is modulated by task difficulty. Clin Neurophysiol. 2010; 121: 98-103.

42) Shin HW, Sohn YH, et al.: Hemispheric asymmetry of surround inhibition in the human motor system. Clin Neurophysiol. 2009; 120: 816-819.

43) Sugawara K, Tanabe S, et al.: Modification of motor cortex excitability during muscle relaxation in motor learning. Behav Brain Res. 2016; 296: 78-84.

44) Pope PA, Holton A, et al.: Cortical control of muscle relaxation: a lateralized readiness potential (LRP) investigation. Clin Neurophysiol. 2007; 118: 1044-1052.

45) Terada K, Ikeda A, et al.: Movement-related cortical potentials associated with voluntary muscle relaxation. Electroencephalogr Clin Neurophysiol. 1995; 95: 335-345.

46) Toma K, Honda M, et al.: Activities of the primary and supplementary motor areas increase in preparation and execution of voluntary muscle relaxation: an event-related fMRI study. J Neurosci. 1999; 19: 3527-3534.

47) Rothwell JC, Higuchi K, et al.: The offset cortical potential: an electrical correlate of movement inhibition in man. Mov Disord.
$1998 ; 13: 330-335$.

48) Buccolieri A, Avanzino L, et al.: Relaxation in distal and proximal arm muscles: a reaction time study. Clin Neurophysiol. 2003; 114: 313-318.

49) Schieppati $M$ and Crenna P: From activity to rest: gating of excitatory autogenetic afferences from the relaxing muscle in man. Exp Brain Res. 1984; 56: 448-457.

50) Schieppati M, Nardone A, et al:: Modulation of the Hoffmann reflex by rapid muscle contraction or release. Hum Neurobiol. 1986; 5: 59-66.

51) Sugawara K, Tanabe S, et al.: Temporal facilitation prior to voluntary muscle relaxation. Int J Neurosci. 2009; 119: 442-452.

52) Suzuki T, Sugawara K, et al.: Excitability changes in primary motor cortex just prior to voluntary muscle relaxation. J Neurophysiol. 2015; 113: 110-115.

53) Sugawara K, Tanabe $\mathrm{S}$, et al.: Functional plasticity of surround inhibition in the motor cortex during single finger contraction training. Neuroreport. 2012; 23: 663-667.

54) Liepert J, Classen J, et al.: Task-dependent changes of intracortical inhibition. Exp Brain Res. 1998; 118: 421-426.

55) Li ZM, Zatsiorsky VM, et al.: Contribution of the extrinsic and intrinsic hand muscles to the moments in finger joints. Clin Biomech (Bristol, Avon). 2000; 15: 203-211.

56) Schneider C, Devanne H, et al.: Neural mechanisms involved in the functional linking of motor cortical points. Exp Brain Res. 2002; 146: 86-94.

57) Slobounov S, Johnston J, et al.: The role of sub-maximal force production in the enslaving phenomenon. Brain Res. 2002; 954: 212-219. 